\title{
Detection of COVID-19 Cases using Histogram of Oriented Gradient (HOG) with Support Vector Machine (SVM) Classifier
}

\author{
Reda Elbarougy \\ Department of \\ Information Technology \\ Faculty of Computer and \\ Artificial Intelligence, \\ Damietta University \\ New Damietta, Egypt
}

\author{
G.M. Behery \\ Department of Computer \\ Science \\ Faculty of Computer and \\ Artificial Intelligence, \\ Damietta University New \\ Damietta, Egypt
}

\author{
Y.M. Younes \\ Department of \\ Mathematics \\ Faculty of Sciences, \\ Damietta University \\ New Damietta, Egypt
}

\author{
Esmail Aboghrara \\ Department of Computer \\ Science \\ Faculty of Sciences, \\ Sebhau University \\ Sebhau, Libya
}

\begin{abstract}
COVID-19 is now considered the most severe and fatal human illness caused by a novel coronavirus. The coronavirus which is considered to have originated in Wuhan, China spread fast around the world in December 2019 and caused a huge the number of fatalities. The early discovery of COVID19 out of precise analysis, specifically in circumstances where there are no immediately visible symptoms, could lower the patient's risk of death. The demand for supplemental diagnostic equipment has grown because there are no precise and available toolkits for automation. However, recent studies using radiological imaging techniques have revealed important information for detecting the COVID19.Combining artificial intelligence and radiological imaging techniques can help improve disease recognition accuracy. A machine learning (ML) strategy for recognizing COVID-19 in chest $\mathrm{x}$-ray images is proposed in this paper.Features were extracted using the histogram-oriented gradient (HOG) from $\mathrm{x}$-ray images. The classification performance of the support vector machine (SVM) classifier used in this study was excellent. The proposed HOG feature technique provided high accuracy reach $(96.6 \%)$.
\end{abstract}

\section{Keywords}

Coronavirus, Histogram-oriented gradient, COVID-19, Chest $\mathrm{x}$-ray, Machine learning, Radiology images

\section{INTRODUCTION}

The Presentation of COVID-19, which started on December 31,2019 , with a report in Wuhan, Hubei Province, China, of pneumonia with unclear causes, has quickly spread to the point of being a pandemic [1], [2], and [3]. COVID-19 is the name of the illness, and the main virus is known as SARSCoV-2. In just 30 days, beginning in Wuhan, this new virus spread across most of China. [4,5].Humans have been infected with the Middle East respiratory syndrome Coronavirus (MERS-CoV), which has caused serious respiratory illness and death[6]. Sore throat, headache, fever, cough, and muscle soreness are common COVID-19 symptoms, and exhaustion, as well as shortness of breath [7].Controlling the spread of COVID-19 requires effective patient screening. Reverse Transcription Polymerase Chain Reaction (RT-PCR) analysiswas utilized to genetically identify SARS-CoV-2, has been the gold standard screening method thusfar. However, it only has a $30 \%$ to $60 \%$ positive rate[8].
Chest radiography imaging, for instance an $\mathrm{x}$-ray or a computed tomography (CT) scan, can be used to diagnose SARS-CoV-2 virus infection.For individuals who acquire serious infections such as pneumonia, indicators such as bilateral, peripheral ground-glass opacities and pulmonary consolidations are an excellent supplemental screening tool.The symptoms of viral infection, COVID-19 pneumonia, on the other hand, might be modest, making it difficult for radiologists to tell it apart from other pneumonias or normal instances.As a result, computer-assisted diagnostic tools capable of detecting COVID-19 pneumonia in chest radiography pictures are in high demand.According to Yoon et al. [9], one out of every three patients had a single nodular opacity in the left lower lung region, while the other two had four or five irregular opacities in both lungs.In most of the patients, Zhao et al. [10] identified ground-glass opacities (GGO) or mixed GGO, as well as lesion consolidation and vascular dilatation.In COVID-19 patients, GGO and consolidation, interlobular septal thickening, and air bronchi gramme ogram were all typical CT findings according to $\mathrm{Li}$ and Xia [11].Another result [9] was that $50 \%$ to $75 \%$ of patients had both lungs were affected by a peripheral focal or multifocal GGO.

In Italy and other nations, chest $\mathrm{x}$-rays are used as a first-line diagnostic technique.Using radiological scans, the health of the lungs, as well as the various stages of disease or recovery, may be precisely identified [13]. According to radiologists, COVID-19 patients' radiological scans revealed a variety of anomalies.COVID-19 characteristics such bilateral GGO and bilateral and multifocal GGO with consolidation are exhibited in chest $\mathrm{x}$-ray images in Fig. 1.

Chest $\mathrm{x}$-ray is used diagnostic in most clinical settings; it takes less time to prepare patients and provides quick results. As a result, chest x-ray can be used to recognize the disease, and allocate medical resources.

Machine learning (ML)has been demonstrated to assist image processing applications such as segmentation, classification, and image analysis.In the medical imaging field, ML algorithms have been used to drastically improve image processing performance [14], [15]. 


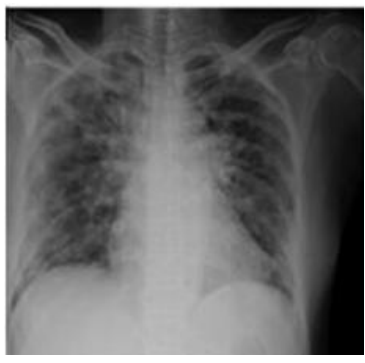

(a)

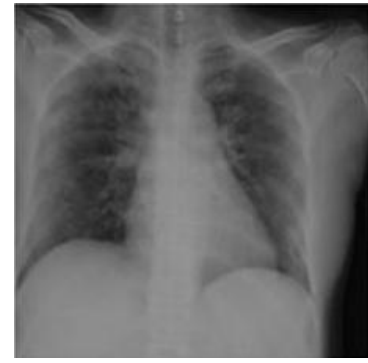

(b)
Fig. 1:Examples of chest x-rays outcomes (a) GGO and (b) GGO with consolidation.

Microscopy photos [16], brain tumor categorization [17], MRI images [18], and retinal images [19] are only a few of the applications of ML.Image classification is done by utilizing a descriptor to extract the essential characteristics from the images, and then using classifiers like support vector Machine (SVM)to use these features in the classification process.

Razzak et al. [3] employed CNN to identify COVID-19 and achieved recognition rate of $98.9 \%$ for binary detection and 94.4 percent for multi classification tests, respectively.Kumar and Kumari [4] used VGG16 and VGG19 to extract COVID19 features, and the overall accuracy of the SVM classifier was 92.7 percent and 92.9 percent, respectively.

In this work, we trained a model based on Histogram-Oriented Gradient (HOG) method for feature extraction using a dataset of COVID-19 pneumonia, viral pneumonia, and normal individuals.To gain the highest performance in categorizing, SVMclassifier for chest $\mathrm{x}$-rayimages is constructed.We can swiftly screen patients who are suspected of having COVID19 pneumonia using the indicated methods.It can help clinicians not only test for COVID-19 more effectively, but also decide on a treatment strategy and establish a therapy plan depending on the infection's source.

\section{MATERIALS AND METHODS}

We used $\mathrm{x}$-ray images in this study to detect coronavirus infected individuals using HOG feature plus SVM classifierbased technique. Because deep learning-based classifiers require a big dataset for training and validation, SVM was utilized instead. Based on the retrieved HOG characteristics, the SVM distinguishes between images of corona-affected $\mathrm{x}$ rays and non-corona-affected $\mathrm{x}$-rays.Medical experts may use this method to identify between COVID-19 patients, pneumonia patients, and healthy people.SVM was tested for COVID-19 identification using the HOG characteristics.

In the next part, the dataset and suggested approach for feature extraction and classification are explained in further depth.

\subsection{Description of the dataset}

A variety of COVID-19 datasets have been pooled, all of which may be seen on the open-source GitHub repository[20]. The overall number of images in the collected dataset of chest $\mathrm{x}$-ray images is 3886.As indicated in Table 1, the first class has 1200 images of a COVID-19 infected individual, the second class contains 1341 images for normal patients, and the third class contains 1345 images of viral pneumonia.Each image of size $227 \times 227$ pixels.
Table 1: The class of the images that were utilized in the dataset that was chosen.

\begin{tabular}{|c|c|c|c|c|}
\hline Class & COVID & Normal & $\begin{array}{c}\text { Viral } \\
\text { pneumonia }\end{array}$ & $\begin{array}{c}\text { Total } \\
\text { images }\end{array}$ \\
\hline $\begin{array}{c}\text { Number } \\
\text { of } \\
\text { images }\end{array}$ & 1200 & 1341 & 1345 & 3886 \\
\hline
\end{tabular}

Gray levels, patch size, dimensions, and other aspects of the $\mathrm{x}$-ray pictures were all varied.Figure 2 shows an example of an x-ray picture of a COVID-19 infected and healthy patient utilized in the study.

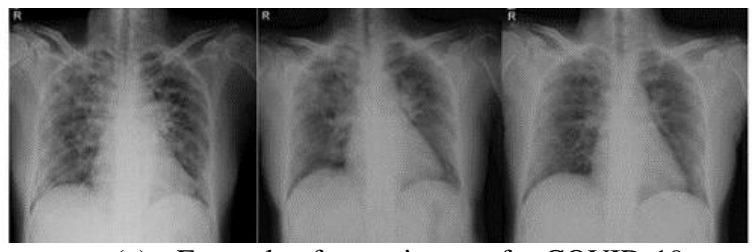

(a) Example of x-ray images for COVID-19 Infected Patient

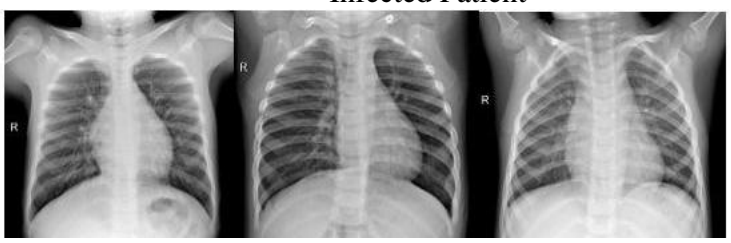

(b) Example of $\mathrm{x}$-ray for Normal Patient

Fig. 2: Examples of chest $x$-rayimages for (a)infected patients of COVID-19 and (b) normal patients.

\subsection{Feature extraction using HOG}

The HOG feature descriptor approach is described by Dalal and Triggs [21]. A HOG approach is used to characterize the gradient orientation in small areas of an image, and the data from all regions is then combined into a single vector.As illustrated in Fig. 3, the HOG feature extraction technique is split down into many steps.

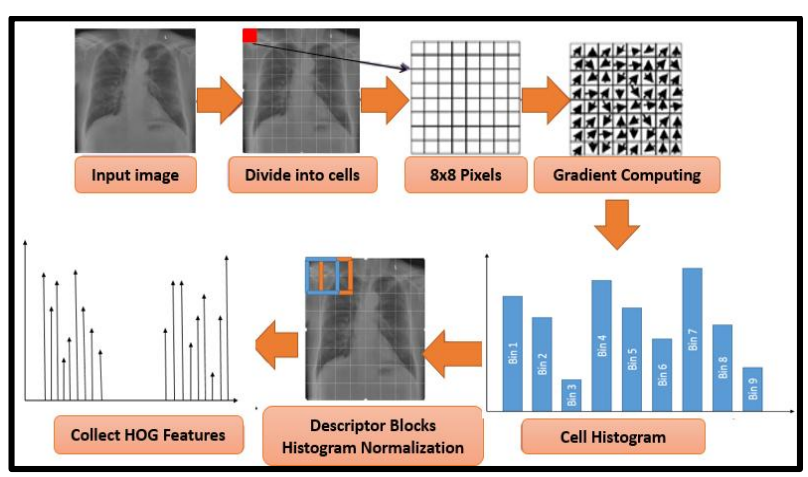

Fig. 3: Steps of HOG feature extraction process

HOG is used to extract features from infected COVID-19 patients as well as normal x-ray pictures with a positive and negative area. HOG has several stages as follows:

Step1:the $1^{\text {st }}$ stepthe input image is distributed into smallerrelated sub-images called cells. Each cell is $8 \times 8$ pixels in size.

Step 2:the $2^{\text {nd }}$ step is used to calculate bothmagnitude and orientation of the gradient for every singlepixel in the cell. In both the horizontal and vertical directions, the most efficient method is to employ a 1-D centeredpoint discrete derivative 
$D_{x}$ and $D_{y}$.Using convolution operation, the horizontal $\left(I_{x}\right)$ and vertical $\left(I_{y}\right)$ derivatives are obtained, see Eqs. (1. and 2.).

$$
\begin{gathered}
I_{x}=I * D_{x} \text { where } D_{x}=[-1,0,1](1) \\
I_{y}=I * D_{y} \text { where } D_{y}=[-1,0,1](2)
\end{gathered}
$$

Eq. 1. means that $I_{\chi}=I(x+1, y)-I(x-1, y)$, while Eq. 2. means that $I_{y}=I(x, y+1)-I(x, y-1)$.For each pixel $\mathrm{I}(\mathrm{x}, \mathrm{y})$, The gradient's magnitude is expressed bythe given Eq. 3.

$$
|G(x, y)|=\sqrt{I_{x}^{2}+I_{y}^{2}}(3)
$$

The direction and the orientation of the gradient are given by the Eq. 4.

$$
\theta(x, y)=\tan ^{-1} \frac{I_{y}}{I_{x}}
$$

Step 3:the $3^{\text {rd }}$ step is to calculate the histograms of each cell. The region of interest (ROI) is classified into small area, called cells. The cell is $8 \times 8$ pixels in size. Each pixel in the cell contributes for a histogram channel based on orientation with a weighted value. The pixel contribution can be represented by the gradient magnitude.The gradient magnitude value is used as a vote weight in [3]. When the gradient is unsigned, the histogram's channels are evenly spaced from $0^{\circ}$ to $180^{\circ}$, while when the gradient is signed, the channels are spaced from $0^{\circ}$ to $360^{\circ}$. The orientation bins in this study are evenly spaced from $0^{\circ}$ to $180^{\circ}$ and are provided by nine of $20^{\circ}$, as shown in Table2. One cell has a feature vector of 9 dimensions. Each bin is contributed by adding the gradient magnitude in the bin corresponding to the gradient direction.

\section{Table2: The orientation bins}

\begin{tabular}{|c|c|}
\hline Bin number & Direction \\
\hline 1 & $0^{\circ}-20^{\circ}$ \\
\hline 2 & $20^{\circ}-40^{\circ}$ \\
\hline 3 & $40^{\circ}-60^{\circ}$ \\
\hline 4 & $60^{\circ}-80^{\circ}$ \\
\hline 5 & $80^{\circ}-100^{\circ}$ \\
\hline 6 & $100^{\circ}-120^{\circ}$ \\
\hline 7 & $120^{\circ}-140^{\circ}$ \\
\hline 8 & $140^{\circ}-160^{\circ}$ \\
\hline 9 & $160^{\circ}-180^{\circ}$ \\
\hline
\end{tabular}

Finally, each cell $C_{i}$ has 9 features as follows: $C_{i}=$ $\left[h_{1}^{i}, h_{2}^{i}, \ldots, h_{9}^{i}\right]$ where $\mathrm{i}$ is the cell number.

Step 4:the $4^{\text {th }}$ step is to calculate descriptors for each block. Gradient strengths vary widely because of local differences in illumination and contrast. To avoid changes in light and contrast can be accomplished by combining the cells into bigger, spatially related blocks. The block is $2 \times 2$ cells in size. These blocks frequently overlap, implying that each cell makes numerous contributions to the final feature. Several studies have found that every four cells form one block, as shown in Fig.4.For each block the descriptors are includes $4 \times 9$ featurei.e.36-component vector given by eq. 5 .

$$
V=\left[a_{1}, a_{2}, \ldots . a_{36}\right](5)
$$
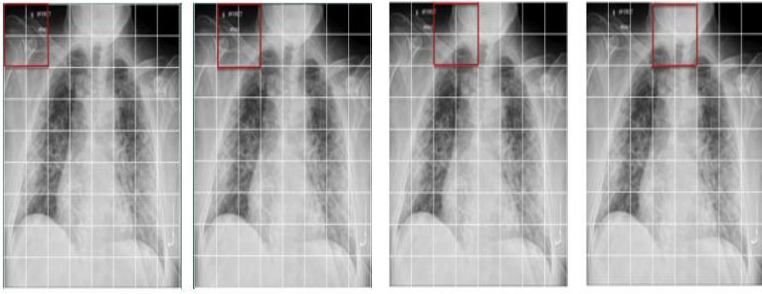

Fig. 4. image segmentation into block of size $2 \times 2$

The gradient local normalization is the next stage. After calculating the HOG features for each cell, the arrays were combined into a single 36-component array and normalized. The vector $\mathrm{V}$ is normalized by dividing all its values by $\mathrm{k}$, which is determined by eq. 6 .

$$
k=\sqrt{\left(a_{1}\right)^{2}+\left(a_{2}\right)^{2}+\cdots+\left(a_{36}\right)^{2}}
$$

The is normalized vector is obtained as in Eq. 7.

$$
|V|=\left(\frac{a_{1}}{k}, \frac{a_{2}}{k}, \ldots \ldots, \frac{a_{36}}{k}\right)(7)
$$

Step 5:in the $5^{\text {th }}$ step theimage's HOG features are calculated in the last stage. The HOG descriptor is calculated for the whole image in one vector that contains the components of all the block regions' normalized cell histograms.An image $227 \times 227$ pixels, contains $27 \times 27$ blocks i.e., the total number of blocks is 729 with variousoverlay. Finally, we construct the normalized vectors acquired form all blocks in a single vector of size $729 \times 36$ features, that represents the final HOG features for one image. Therefore, we obtain 26,244features for each image. A graphical representation for an example of theobtained the features of full image as show in fig. 5 .
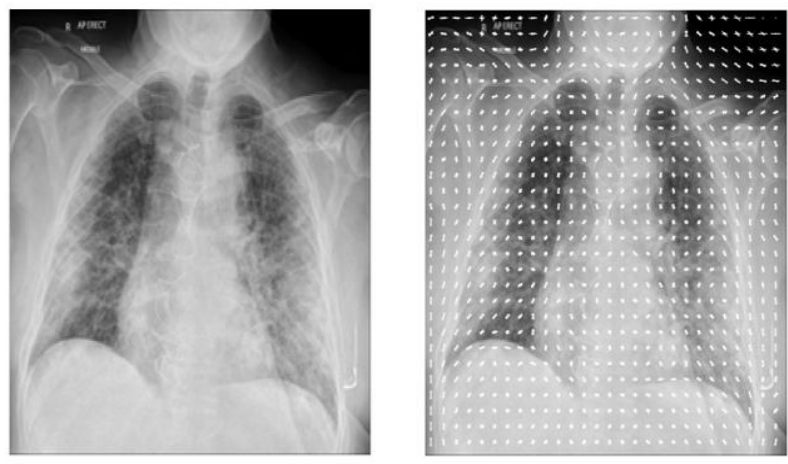

Fig. 5: (Left) An input image. (Right) HOG features of input image.

\subsection{Classification}

The SVM approach was chosen for this study because it is commonly used for classification issues and can effectively execute numerous classification procedures; also, in terms of classification performance, it outperforms other machine learning algorithms such as K-nearest neighbor.SVMhave been used after the feature extraction step. However, this is the final stage in the disease recognition process, where the disease is recognized based on the feature set extraction from the HOG.SVM is a supervised learning method that may be employed to solve classification and regression issues. It is most employed in classification problems due to its efficacy 
and high accuracy in many fields. It is of two types: binary classification, where it classifies only two groups, and multiclass classification, where it classifies more than one group. The SVM algorithm's main goal is to determine the optimal separating hyperplane between classes. In this study SVM classifier was used for classification. This classifier is based on statistical learning theory. Given training data:

$\omega^{\mathrm{T}} \cdot x=0$

Where $\omega$ and $x$ represent vectors and $\omega^{\mathrm{T}} \cdot x$ represent the dot product of these two vectors, usually the $\omega$ vector iscalled a vector of weights. Our training data can be represented mathematically as follows:

$\left\{\left(x_{1}, \mathrm{y}_{1}\right),\left(x_{2}, \mathrm{y}_{2}\right), \ldots \ldots,\left(x_{\mathrm{n}}, \mathrm{y}_{\mathrm{n}}\right)\right\} \in \mathrm{R}^{\mathrm{n}}$

It means that our data is represented by a pair of data $\left(x_{\mathrm{n}}, \mathrm{y}_{\mathrm{n}}\right)$, where $x_{\mathrm{n}}$ represents the feature vector which has $\mathrm{n}$ dimensions, and $y_{n}$ represents the label of $x_{\mathrm{n}}$. When $\mathrm{y}_{\mathrm{i}}=1$, then this means that the data belongs to group 1 , and if $y_{i}=-1$ then it belongs to group -1 .

\section{RESULTS AND DISCUSSION}

This study employed a dataset of 3886 chest $\mathrm{x}$-ray pictures for training-set, testing-set, and validation-set as indicated in Table 1, to confirm the proposed method for recognizing COVID-19.To address the issue of data inconsistency, the data distribution was laminated in this study. The validation photographs were brought from the training set, while the testingset was shot prior.

This study employed 657 normal pictures, 588 COVID-19positive images, and 659 viral pneumonias for training. In addition, the testing included 402 normal pictures, 360 COVID-19-positive images, and 404 viral pneumonias.The training dataset does not include these testing photos. As demonstrated in Table 3, this system had an extra 816 validation images for the normal, COVID-19, and viral pneumonia image classes.

Table 3:The number of training, validation, and testing instances used.

\begin{tabular}{|c|c|c|c|c|}
\hline & $\begin{array}{c}\text { Viral } \\
\text { pneumonia }\end{array}$ & normal & COVID & $\begin{array}{c}\text { Total } \\
\text { images }\end{array}$ \\
\hline Train & 659 & 657 & 588 & 1904 \\
\hline Validation & 282 & 282 & 252 & 816 \\
\hline Test & 404 & 402 & 360 & 1166 \\
\hline Total & 1345 & 1341 & 1200 & 3886 \\
\hline
\end{tabular}

Table 4 shows the confusion matrix derived from all the systems' assessed performance attributes during the classification stage.The suggested technique requires labelled test data to confirm its predicted output during the evaluation.The system's total performance is depicted by the confusion matrix.The system properly detects 346 of the 360 COVID photos and fails to identify 14 of them. Furthermore, the system correctly finds 382 normal photographs out of 402 , while incorrectly identifying 20 normal shots. Finally, the system correctly detects 381 viral pneumonia photographs out of 404 , while failing to detect 22 images.
Table4: Confusion matrix

\begin{tabular}{|c|c|c|c|c|}
\hline Priginal & COVID & normal & $\begin{array}{c}\text { Viral } \\
\text { pneumonia }\end{array}$ & Total \\
\hline COVID & 346 & 4 & 10 & 360 \\
\hline normal & 1 & 382 & 19 & 402 \\
\hline $\begin{array}{c}\text { Viral } \\
\text { pneumonia }\end{array}$ & 3 & 19 & 381 & 403 \\
\hline
\end{tabular}

The overall accuracy of the proposed system is $96.6 \%$ which ensures that true results, either true positive or true negative, are high. The proposed method is suitable also for real-time applications. The processing time was about 0.56 seconds on a machine equipped with a core i7 processor and $8 \mathrm{~GB}$ of RAM. This makes the proposed method reliable for clinical application.

\section{CONCLUSIONS}

The coronavirus COVID-19 pandemic began with an infected person who had a life-threatening health condition and soon spread over the world. The application of machine learning techniques to detect COVID-19 will help patients recover more quickly throughout the world.This study presents a COVID-19 identification approach that uses chest $\mathrm{x}$-ray images to detect whether a person is infected.To begin, a database of 3886 chest $\mathrm{x}$-ray images is gathered, divided into three categories: COVID-19, normal, and viral pneumonia. Then, from all the photos, extracting theHOG features.Finally, an SVM classifier is utilized to categorize chest x-ray images, with the HOG characteristics as the input and the illness type as the output. The results of the evaluation show that the proposed system has a very high accuracy of 96.6 percent.

This research may be further optimized in a variety of ways. We'll use Convolutional neural network $(\mathrm{CNN})$ for feature extraction to see if we can enhance the detection rate. Furthermore, a feature selection strategy could be used to minimize the computing time, making COVID-19 detection acceptable for real-time applications.

\section{REFERENCES}

[1] Bull, F. C., Al-Ansari, S. S., Biddle, S., Borodulin, K., Buman, M. P., Cardon, G., ... \&Willumsen, J. F. (2020). World Health Organization 2020 guidelines on physical activity and sedentary behaviour. British journal of sports medicine, 54(24), 1451-1462.

[2] Wu, F., Zhao, S., Yu, B., Chen, Y. M., Wang, W., Song, Z. G., ... \& Tian, J. H. (2020). Pei 465 YY et al: A new coronavirus associated with human respiratory disease in 466. China. Nature, 579(7798), 265-269.

[3] Yang, X., Yu, Y., Xu, J., Shu, H., Liu, H., Wu, Y., ... \& Shang, Y. (2020). Clinical course and outcomes of critically ill patients with SARS-CoV-2 pneumonia in Wuhan, China: a single-centered, retrospective, observational study. The Lancet Respiratory Medicine, 8(5), 475-481.

[4] Wu, Z., \& McGoogan, J. M. (2020). Outbreak in China: summary of a report of 72314 cases from the Chinese center for disease control and prevention. JAMA, 323(10.10), 1001

[5] Holshue, M. L., DeBolt, C., Lindquist, S., Lofy, K. H., 
Wiesman, J., Bruce, H., ... \& Pillai, S. K. (2020). First case of 2019 novel coronavirus in the United States. New England Journal of Medicine.

[6] Kong, W., \& Agarwal, P. P. (2020). Chest imaging appearance of COVID-19 infection. Radiol Cardiothoracic Imaging 2 (1): e200028.

[7] Hu, Z., Ge, Q., Li, S., Jin, L., \&Xiong, M. (2020). Artificial intelligence forecasting of covid-19 in china. arXiv preprint arXiv:2002.07112.

[8] Zu, Z. Y., Jiang, M. D., Xu, P. P., Chen, W., Ni, Q. Q., Lu, G. M., \& Zhang, L. J. (2020). Coronavirus disease 2019 (COVID-19): a perspective from China. Radiology, 296(2), E15-E25.

[9] Jiang, F., Jiang, Y., Zhi, H., Dong, Y., Li, H., Ma, S., ... \& Wang, Y. (2017). Artificial intelligence in healthcare: past, present and future. Stroke and vascular neurology, 2(4).

[10] Deo, R. C. (2015). Machine learning in medicine. Circulation, 132(20), 1920-1930.

[11] Sarker, I. H., Kayes, A. S. M., \& Watters, P. (2019). Effectiveness analysis of machine learning classification models for predicting personalized context-aware smartphone usage. Journal of Big Data, 6(1), 1-28.

[12] Mahdy, L. N., Ezzat, K. A., Elmousalami, H. H., Ella, H. A., \&Hassanien, A. E. (2020). Automatic x-ray covid-19 lung image classification system based on multi-level thresholding and support vector machine. Med Rxiv.

[13] Vickers, N. J. (2017). Animal communication: when i'm calling you, will you answer too? Current biology, 27(14), R713-R715.

[14] Litjens, G., Kooi, T., Bejnordi, B. E., Setio, A. A. A., Ciompi, F., Ghafoorian, M., ... \& Sánchez, C. I. (2017).
A survey on deep learning in medical image analysis. Medical image analysis, 42, 60-88.

[15] Altaf, F., Islam, S. M., Akhtar, N., \& Janjua, N. K. (2019). Going deep in medical image analysis: concepts, methods, challenges, and future directions. IEEE Access, 7, 99540-99572.

[16] Xing, F., Xie, Y., Su, H., Liu, F., \& Yang, L. (2017). Deep learning in microscopy image analysis: A survey. IEEE transactions on neural networks and learning systems, 29(10), 4550-4568.

[17] Muhammad, K., Khan, S., Del Ser, J., \& de Albuquerque, V. H. C. (2020). Deep learning for multigrade brain tumor classification in smart healthcare systems: A prospective survey. IEEE Transactions on Neural Networks and Learning Systems, 32(2), 507-522.

[18] Liu, J., Pan, Y., Li, M., Chen, Z., Tang, L., Lu, C., \& Wang, J. (2018). Applications of deep learning to MRI images: a survey. Big Data Min Anal 1 (1): 1-18.

[19] Seeböck, P., Orlando, J. I., Schlegl, T., Waldstein, S. M., Bogunović, H., Klimscha, S., ... \& Schmidt-Erfurth, U. (2019). Exploiting epistemic uncertainty of anatomy segmentation for anomaly detection in retinal OCT. IEEE transactions on medical imaging, 39(1), 8798.

[20] Cohen, J. P., Morrison, P., Dao, L., Roth, K., Duong, T. Q., \&Ghassemi, M. (2020). Covid-19 image data collection: Prospective predictions are the future. arXiv preprint arXiv:2006.11988.

[21] Dalal, N., \&Triggs, B. (2005, June). Histograms of oriented gradients for human detection. In 2005 IEEE computer society conference on computer vision and pattern recognition (CVPR'05) (Vol. 1, pp. 886-893). Ieee. 